\title{
Focal Molography: Coherent Microscopic Detection of Biomolecular Interaction
}

\author{
Christof Fattinger* \\ Roche Pharmaceutical Research and Early Development, Discovery Technologies, \\ Roche Innovation Center Basel, F. Hoffmann-La Roche Ltd., Grenzacherstrasse 124, 4070 Basel, Switzerland \\ (Received 29 July 2013; revised manuscript received 5 May 2014; published 11 August 2014; corrected 17 October 2014)
}

\begin{abstract}
We introduce and theoretically investigate here a novel analytical method that we have called focal molography, in which molecular interactions are made visible through scattering of coherent light by a coherent pattern of molecules. The scattered light quantifies the presence of molecules at molecular interaction sites. It is separated from noncoherent background scatter by a combination of local dark-field illumination, interference enhancement, and spatial filtering. The latter is achieved by holographic focusing of the wave field generated by the coherently assembled molecules onto an Airy disk and by subtraction of the noncoherent irradiance in the focal plane outside the disk from the irradiance in the disk. This new microscopic method allows distinct detection of low-refractive-index contrast in the nanoenvironment of biomolecules from which information on the interaction of the coherently assembled molecules with molecules in a liquid or gaseous sample may be deduced. The noncoherent surroundings of the coherently assembled molecules consist of freely diffusing solvent and solute molecules. The surroundings, as well as changes in temperature, do not contribute to the coherent signal in the diffraction focus. Interference lithography or high-resolution-imaging lithography can be used to synthesize the coherent pattern of molecules on a monolithic substrate. The coherent pattern of molecules constitutes a synthetic phase hologram that creates a diffraction-limited light wave. We suggest the term "mologram" for the coherent assembly of functional nanostructures and the term "focal molography" for label-free or labeled analysis of molecular interactions through the measurement of the properties of light in the focus of the mologram. We derive analytical formulas that express the detection signal and the sensitivity of focal molography on the surface of a high-refractive-index thin-film optical waveguide in terms of known parameters. We discuss the implementation of a readout system for molograms on a thin-film optical waveguide by adapting a confocal laser-scanning microscope to a bifocal laser-scanning microscope.
\end{abstract}

DOI: 10.1103/PhysRevX.4.031024

\section{INTRODUCTION}

Phase contrast in transmitted light, discovered by Frits Zernike in 1934, revolutionized the biomedical research of living—i.e., unstained—cells [1]. The observation of polarizable molecular structures under the microscope made it possible to investigate subcellular components and the interaction of biological macromolecules (e.g., chromosomes) in their natural biological surrounding medium without the use of stains or labels [2]. In phase contrast $(\mathrm{PhC})$, "direct light" and "diffracted light" from the same phase object are brought to interference [1,2]. PhC converts a small spatial phase modulation in the microscopic object into a visible intensity modulation, and, in this way,

\footnotetext{
*christof.fattinger@roche.com
}

Published by the American Physical Society under the terms of the Creative Commons Attribution 3.0 License. Further distribution of this work must maintain attribution to the author $(s)$ and the published article's title, journal citation, and DOI.
Subject Areas: Biological Physics,

Interdisciplinary Physics, Optics formerly invisible biological structures appear in dark or light contrast to the surroundings.

Digital holographic microscopy (DHM) in transmission mode is dedicated to the quantitative noninvasive investigation of refractive-index contrast or optical path length in three-dimensional phase objects, such as living cells in culture $[3,4]$. The DHM instrument is basically a MachZehnder interferometer. A microscope objective collects direct light and diffracted light from the specimen and forms the object wave, which interferes, in off-axis geometry, with a reference wave to produce hologram intensity [3]. DHM uses numerical processing of the complex wave front in the digitally recorded hologram to compute small changes of phase contrast or optical path length in the specimen $[3,4]$.

$\mathrm{PhC}$ and DHM enable the analysis of phase information produced by transparent specimens in high-resolution light microscopy. PhC and DHM allow one to study molecular interactions that can be measured through the detection of changes in the polarizability of the molecules in living cells. The optical manifestation of molecular processes in subcellular structures can be detected without the need for 
stains or fluorescent labels. PhC and DHM do not allow the detection of the extremely small changes in refractive-index contrast that accompany the interactions of biomolecules in a sparse pattern of separated molecules. Noninvasive investigation of biological specimens strives for new ways and means that allow one to probe the phase contrast in transparent microscopic specimens and to examine minuscule changes in phase contrast induced by biomolecular processes.

The small spatial phase modulation that accompanies the recognition and binding of molecules to immobilized biomolecules in a thin layer on the surface of a chip can-in principle - be measured either by direct light or by diffracted light. It is commonly believed that diffractometric optical methods for the label-free detection of biomolecular interactions cannot do any better than at present; i.e., they have reached their physical limit. We demonstrate here that this presumption does not apply. To help clarify our argument, a brief review of the current situation is first presented.

The advent of sensitive refractometric optical biosensors in the early 1990s [5,6] enabled the interactions between biomolecules to be studied in much greater detail. In the last two decades, a wide range of transducer principles was developed to enable the direct monitoring of noncovalent biospecific interactions between unlabeled biomolecules in real time [7]. Refractometric optical biosensors measure the resonance shift or the phase shift of a surface wave (surface plasmon or waveguide mode) $[7,8]$. Label-free biomolecular interaction analysis with optical biosensors requires the immobilization of one interaction partner (the "receptor," in the parlance of this paper) in a thin layer on the sensor surface to which a complementary interaction partner (the "ligand") may bind. The layer forms a thin phase object that spreads over lateral dimensions of $L$ with $L \gg \lambda$, and $\lambda$ is the vacuum wavelength of the optical surface wave. The thickness $t_{m}$ of the phase object on the supporting surface is small $\left(t_{m}<\lambda\right)$, possibly as small as approximately $\lambda \times 10^{-6}$ [8]. Values of $t_{m}$ below approximately $0.1 \mathrm{~nm}$ resemble the averaged thickness of a submonolayer [8]. The surface wave propagating along the thin phase object is subjected to a phase retardation that quantifies the concentration of ligand molecules at the binding sites of the immobilized receptor $[9,10]$.

Refractometric optical biosensors are used for the characterization of molecular interactions in terms of both affinity and chemical kinetics as well as for the concentration analysis of a ligand in a sample that is brought into contact with the sensor surface $[5,6,10]$. Since its introduction in 1993, surface plasmon resonance (SPR) evolved to the gold standard for measuring the kinetics of biomolecular interactions, i.e., the rates of complex formation $\left(k_{a}\right)$ and dissociation $\left(k_{d}\right)$ [10-14]. In drug discovery, optical biosensors can also detect multiple sequential interactions during the formation of a complex involving more than two interaction partners $[10,14]$. Complex formation on the sensor surface can be monitored as each component is incorporated into a multimolecular complex [10,14].

The refractive index of a submonolayer of surface-bound biomolecules [15] and the refractive index of a thin hydrogel layer with subwavelength thickness $t_{m}$ to which a receptor is immobilized can be measured precisely with refractometric optical biosensors; see, e.g., Refs. [16-19]. The change of the refractive index of the thin phase object on the supporting surface during a binding reaction and its relation to the surface mass density of the ligand that binds to the immobilized biomolecules is well known and extensively discussed in the literature on optical biosensors; see, e.g., Refs. $[18,19]$.

Diffractometric optical biosensors allow the label-free detection of binding events on a surface by measuring the intensity of light diffracted at the sensor surface in a variety of optical configurations; see, e.g., Refs. [20,21] and references therein.

Refractometric [22-24] and diffractometric [25] optical biosensors may be applied to sandwich assays that are optionally amplified by secondary mass tags (latex or gold nanoparticles). The labeled detection of a sandwich complex enhances the sensitivity for the detection of the ligand on the sensor surface by several orders of magnitude as compared to the corresponding label-free assay $[22,23]$.

Because it is hard to exclude the nonbiospecific binding of high-abundance proteins in the sample to the thin layer on the sensor surface, even after careful washing, the affinity-based identification of an analyte in serum, plasma samples, and other body fluids is significantly less sensitive in label-free direct-binding assays than labeled detection of the same analyte in a sandwich-type assay. This is the reason why the labeled detection of a sandwich complex is the preferred assay scheme for biodiagnostic immunoassays. The labeled detection of a sandwich complex can only be applied to a known ligand, e.g., the detection of a biomarker in a diagnostic test. It does not allow us to follow the binding process between the receptor and the ligand in real time. In the life sciences, labeled detection is not applicable for the discovery of unknown ligands of a receptor in exploratory investigations of affinity binding [14].

Even though the surface waves [i.e., the transverse electric (TE) mode and the transverse magnetic (TM) mode of the thin-film optical waveguide] of the bidiffractive grating-coupler biosensor described in Refs. [22] and [23] are coupled into and out of the waveguide by diffraction gratings, the measurement of the surface mass density on this sensor surface is purely refractometric. Every change of the refractive index within the penetration depth of the evanescent field is recorded by the bidiffractive grating-coupler biosensor through the measurement of changes in the relative phase between the two coherently excited modes of the thin-film waveguide (TE and TM). 
Comprehensive investigations of the advantages and the disadvantages of the miniaturization of assays based on surface capture are discussed in Refs. [26] and [27]. The fundamental sensitivity limits of biosensors based on surface capture are analyzed in Ref. [27].

\section{A. Limit of detection of refractometric optical biosensors for label-free biomolecular interaction analysis}

Because refractometric optical biosensors use direct light for detecting molecular interactions, all refractive-index changes in the thin layer on the sensor surface (caused by nonbiospecific binding of ligand molecules to the sensor surface or to matrix molecules in the thin layer on the sensor surface, by changes of composition or ionic strength of the supernatant, or by small changes in temperature) contribute to the detected phase signal that is used for the deduction of the time-dependent course of the molecular interactions on the sensor surface.

The accumulated phase shift measured by refractometric optical biosensors (e.g., SPR and thin-film optical waveguide biosensors) depends on the propagation length of the surface wave. The propagation length is limited for SPR but not for waveguides. It is important to note that a given surface mass density results in a larger "specific phase shift" for SPR than for waveguides [9]. The specific phase shift is the phase shift per unit surface mass density and per unit propagation length of the surface wave. The phase shift that is induced by a given surface mass density $\left(\mathrm{pg} / \mathrm{mm}^{2}\right)$ divided by the propagation length of the surface wave is higher for SPR as compared to biosensors based on thinfilm optical waveguides [9]. This is the reason why extensive research on label-free optical biosensors over the last 20 years has not changed the preeminent role of SPR in direct-binding assays [5,6,10-14]. The sensitivity of all refractometric optical biosensors in direct-binding assays is limited by small, unavoidable refractive-index changes that accumulate over the propagation path of the surface wave; it is not limited by the accuracy of the measurement of the total phase shift $[8,9,11,12]$, which may be improved for waveguides by elongating the propagation path of the surface wave $[8,9]$.

The hydrogel layer on the surface of the SPR biosensor consists mainly of water [16-18]. An increase in temperature of $0.02{ }^{\circ} \mathrm{C}$ decreases the refractive index $n_{0}$ of the aqueous solution on the sensor surface by $\Delta n_{0} \approx-2.2 \times 10^{-6}$. (The temperature dependence of the refractive index of water at $T=25^{\circ} \mathrm{C}$ and $\lambda=635 \mathrm{~nm}$ is $d n / d T \approx-1.06 \times$ $10^{-4} 1 /{ }^{\circ} \mathrm{C}$.) The hydrogel layer to which receptor molecules are immobilized is approximately $100 \mathrm{~nm}$ thick [18]. The association of approximately $1.2-\mathrm{pg} / \mathrm{mm}^{2}$ protein with a 100 -nm-thick layer on the sensor surface changes the refractive index $n_{0}$ of the layer by $\Delta n_{0} \approx 2.2 \times 10^{-6}$ $[15,18]$. The phase shift induced by the binding of a small ligand to a relatively large immobilized receptor on the sensor surface is comparable to the phase shift caused by very small temperature instabilities during the measurement $[11,14,18]$. A minute instability in temperature during the measurement can readily override the phase shift measured by refractometric optical biosensors (SPR and thin-film optical waveguide sensors). This is the reason why refractometric optical biosensors need to utilize very precise temperature stabilization during the measurement of binding processes on the sensor surface.

Refractometric optical biosensors probe every change in refractive index in the thin layer on the sensor surface. Any change in composition of the freely diffusing solvent and solute molecules in the thin layer on the sensor surface caused by minute changes of composition or ionic strength of the supernatant contributes to the measured phase signal. This is the reason why highly sensitive label-free biomolecular interaction analysis with refractometric optical biosensors requires a steady flow of buffer solution with stable composition across the sensor surface during the measurement $[5,6,10-14]$.

Nonbiospecific interactions of buffer constituents with the thin layer on the sensor surface can be compensated in the refractometric measurement by referencing the measured phase signal against a thin layer without receptor molecules $[11,12,14]$. Using this referencing-scheme quantification of $\mathrm{pg}$ amounts of ligand molecules per $\mathrm{mm}^{2}$ in the thin layer on the sensor surface is possible with highly sensitive refractometric optical biosensors based on SPR $[11,12,14]$.

\section{B. Limit of detection of diffractometric optical biosensors for label-free biomolecular interaction analysis}

In a recent paper [21], Nolte and co-workers argue that diffraction-based biosensors have a very low sensitivity to detection of the averaged thickness $d$ of small molecules or to thin layers of even larger molecules on the surface of the sensor chip because of the small value of $(d / \lambda)^{2}$ [21]. The new diffraction-based biosensor method described in this paper is able to overcome this deficiency of diffractionbased biosensors for label-free biomolecular interaction analysis by probing the diffracted wave in a diffractionlimited focal spot.

Focal molography provides signal amplification through the coherent addition of the phase signals from the coherently assembled molecules in the mologram by focusing of the phase signal from the coherently ordered molecules into a diffraction-limited focal spot. Focal molography has no apparent intrinsic lower limit of detection in terms of low receptor occupancy. It is shown below that the detection sensitivity of focal molography scales with the square of the diameter of the mologram. It is also shown that the sensitivity of focal molography is not limited by the unavoidable nonbiospecific interactions of solvent and solute molecules with the sensor surface or with matrix molecules in the thin layer on the sensor 
surface. Focal molography is expected to greatly increase the sensitivity and the detection accuracy of real-time labelfree biomolecular interaction analysis.

\section{FOCAL MOLOGRAPHY}

This paper provides the theoretical basis for a new sensing concept, i.e., "focal molography." The new concept has two unique advantages over existing label-free and labeled optical sensing methods: (i) It eliminates the effect of nonbiospecific binding that is the limiting factor in all current state-of-the-art sensing devices and (ii) it has a very high sensitivity that can theoretically be improved nearly without limitation by increasing the size of the sensor. Thanks to these properties, focal molography is expected to open new investigative possibilities in the analysis of affinity binding with high application potential in molecular biology and diagnostics.

\section{A. Physical perceptions of focal molography}

A value for the molecular scattering of light, in the analysis of Lord Rayleigh, may be obtained by summing the scattering from individual molecules (dipole scatterers). This calculation gives the same result as a calculation of scattering from density fluctuations in the scattering medium. We consider now the scattering of coherent light by an isolated receptor-ligand complex. The intensity of near-backward scattering $I_{\text {scat }}$ by an isolated receptor molecule with refractive index $n_{R}$ embedded in a medium with refractive index $n_{0}$ in plane-polarized light is

$$
\begin{aligned}
\frac{I_{\text {scat }}(r)}{I_{R}} & =\frac{9 \pi^{2} n_{0}^{4}}{r^{2} \lambda^{4}}\left|\frac{n_{R}^{2}-n_{0}^{2}}{n_{R}^{2}+2 n_{0}^{2}}\right|^{2} V_{R}^{2} \\
& =\frac{9 \pi^{2} n_{0}^{4}}{r^{2} \lambda^{4}}\left(\frac{d n}{d c}\right)^{2} \frac{M_{R}^{2}}{N_{A}^{2}} \bar{n}\left(\rho_{R}\right),
\end{aligned}
$$

where $r$ is the distance from the molecule to the point of observation, which is large compared to the vacuum wavelength $\lambda$ [28]. $I_{R}$ is the intensity of the plane-polarized field incident on the receptor molecule. The volume of the receptor molecule $V_{R}$ and the index factor $\bar{n}\left(\rho_{R}\right)$ are given by

$V_{R}=\frac{d n}{d c} \frac{M_{R}}{N_{A}\left(n_{R}-n_{0}\right)} ; \quad \bar{n}\left(\rho_{R}\right)=\frac{\left(n_{R}+n_{0}\right)^{2}}{\left(n_{R}^{2}+2 n_{0}^{2}\right)^{2}}$,

where $M_{R}$ denotes the molar mass of the receptor molecule and $N_{A}$ the Avogadro constant. For the refractive-index increment $d n / d c$, we use the common value for proteins in water $0.182 \mathrm{ml} / \mathrm{g}[15,29,30]$; this value is also a fairly good approximation for any biological ligand that may bind to the receptor in water $n_{0}=1.33$. The refractive index of a protein molecule depends on its mass density $\rho_{R}: n_{R}=n_{0}+(d n / d c) \rho_{R}$. The index factor $\bar{n}\left(\rho_{R}\right)$ varies only slightly for different mass densities of the receptor protein in an aqueous medium: The three values of $\rho_{R}=0.6$,
$1,1.35 \mathrm{~g} / \mathrm{ml}$ result in values of $\bar{n}\left(\rho_{R}\right)=0.244,0.238,0.233$, respectively. Thus, the mass of the receptor-ligand complex provides a generally applicable measure for the approximate calculation of the increase in the scattering intensity caused by the binding of a ligand molecule to the receptor molecule. The increase in scattering intensity caused by the binding of a ligand to the receptor is - in the first-order approximationproportional to the square of the molecular masses of the receptor-ligand complex.

In a thought experiment, we consider the coherent scattering by a collection of $N_{R}$ isolated (i.e., separated) protein molecules embedded in a homogeneous medium $n_{0}$. The molecules are assumed to be localized in a thin layer with thickness $t_{m} \ll \lambda$ that forms an ellipsoid of revolution that has two optically conjugated foci $\mathrm{F}$ and $\mathrm{F}^{\prime}$; see Fig. 1. The distance from the first focus $\mathrm{F}$ to the ellipsoid of revolution and back to the second focus $\mathrm{F}^{\prime}$ is $2 r$.

The molecules in the thin layer are irradiated by a diverging laser beam originating from the focus located at F. The coherently scattered light in the near-backward direction is confined to a diffraction spot (Airy disk) centered at $\mathrm{F}^{\prime}$. For a random distribution of the molecules in the thin layer, the diameter $d_{A}$ of the Airy disk is given by Abbe's formula $d_{A}=\lambda /\left(2 n_{0} \sin \alpha\right)$, where $\alpha$ is half the opening angle of the diverging laser beam. We relate the coherently scattered power $P_{\text {scat }}$ incident on the Airy disk at $\mathrm{F}^{\prime}$ to the power $P_{R}\left(\right.$ Disk- $\left.d_{A}\right)$ incident on a second disk with diameter $d_{A}$ located on the ellipsoid of revolution

$$
\frac{P_{\text {scat }}(\text { Airy })}{P_{R}\left(\text { Disk- } d_{A}\right)}=9 \pi^{2} \frac{n_{0}^{4}}{r^{2} \lambda^{4}}\left(\frac{d n}{d c}\right)^{2} \frac{N_{R}^{2} M_{R}^{2}}{N_{A}^{2}} \bar{n}\left(\rho_{R}\right) .
$$

Equation (3) is valid for a collection of $N_{R}$ molecules that form a coherent scattering system. For a given mass of protein in the thin layer, the ratio in Eq. (3) increases with decreasing $r$ in the second power of $(1 / r)$. For the values

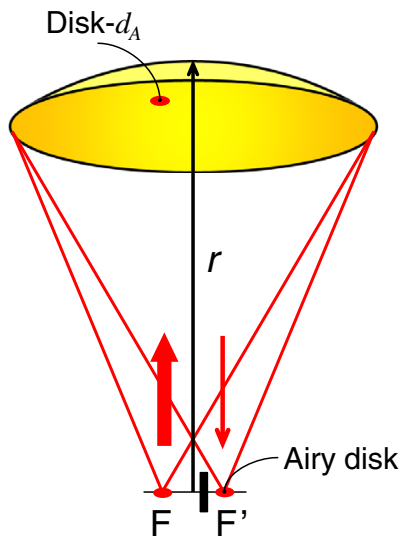

FIG. 1. Coherent scattering by separated biomolecules in an aqueous medium. The molecules are assumed to be localized randomly in a thin layer with thickness $t_{m} \ll \lambda$. The layer forms a part of an ellipsoid of revolution that has two optically conjugated foci $\mathrm{F}$ and $\mathrm{F}^{\prime}$. 
$n_{0}=1.33, r=0.5 \mathrm{~mm}, \lambda=635 \mathrm{~nm}$, and $\rho_{R}=1$, we obtain for $P_{\text {scat }}($ Airy $) / P_{R}\left(\right.$ Disk- $\left.d_{A}\right)=5.4 \times 10^{-5}$ for 1-pg and 54 for 1-ng protein in the thin layer, respectively. The ratio of the incident power densities in Eq. (3) reaches the value 1 for approximately 136-pg protein in the coherent scattering system. This small amount of coherently assembled biological matter irradiates power density on the Airy disk in the focus of the coherent scattering system that equals the power density of the beam of light impinging on the ellipsoid of revolution. The protein molecules are assumed to be randomly distributed within the aperture of the coherent scattering system.

We suggest the term "mologram" for the coherent assembly of functional nanostructures and the term "focal molography" for the analysis of molecular interactions through the measurement of the properties of light in the focus of the mologram.

Direct light from the source focus is shielded off from the detection focus generated by the coherently assembled molecules in the mologram. Direct light can be caused by reflection at optical surfaces and interfaces. In the focal molography configuration depicted in Fig. 1, the source F, the mologram, and the diffraction focus $\mathrm{F}^{\prime}$ are all assumed to be embedded in the same homogeneous medium with refractive index $n_{0}$. This rather special focal molography configuration has no optical surfaces and interfaces; the beam stop between $\mathrm{F}$ and $\mathrm{F}^{\prime}$ shields off direct light. The noncoherent surroundings of the coherently assembled molecules consisting of freely diffusing solvent and solute molecules do not contribute to the coherent signal in the diffraction focus $\mathrm{F}^{\prime}$.

The new method for real-time label-free biomolecular interaction analysis that is described and analyzed in this paper exploits molograms on the surface of a thin-film optical waveguide of high refractive index. The purpose of the waveguide in the detection of the mologram becomes apparent by consideration of the optical properties of the bidiffractive grating coupler previously described [31].

This experimental configuration [31] makes clearly visible the presence of a very shallow surface-relief grating with an amplitude of a few nm or even much less. Without the waveguide located in close proximity to the grating structure, the very shallow grating on the surface of a perfectly transparent substrate can hardly be observed or detected by optical means. The tight confinement of the transversal field distribution of the mode propagating in the dielectric waveguide of high refractive index ensures an effective interaction of the guided wave with the immediate vicinity of the waveguide surface. Observation of the optical properties of the bidiffractive grating coupler guides the author to an intuitive understanding of the physical principles of focal molography.

\section{B. Focal molography on a thin-film optical waveguide}

After consideration of these basic physical principles, we introduce and analyze a new microscopic method for

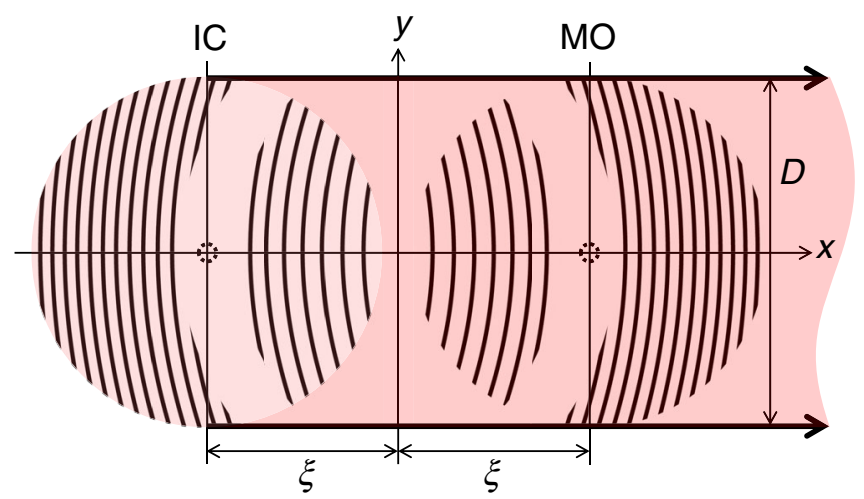

FIG. 2. Grating lines of a 3D-2D-3D optics imaging system with an input coupler (IC) and a mologram (MO). Only every 50th line is shown, together with some adjacent lines.

probing extremely low-refractive-index contrast in a laterally elongated phase object with a thickness $t_{m}<\lambda$; a conceivable realization of it is shown in Figs. 2 and 3.

The investigated molecules are preferentially immobilized along the lines of a phase grating that forms a diffractive lens with diameter $D$ on the surface of a thinfilm waveguide. The coherent pattern of molecules is referred to as a mologram. A diverging laser beam is coupled to the TE mode of the waveguide by an input coupler (focal grating coupler) of diameter $D$. While propagating through the mologram, the guided mode partially decouples from the waveguide through diffraction at the molecules in the mologram and forms a converging beam. The input coupler (IC) and the mologram constitute an integrated 3D-2D-3D optics imaging system with two optically conjugated foci $\mathrm{F}$ and $\mathrm{F}^{\prime}$.

Resonant coupling of the laser beam to a thin-film waveguide of high refractive index enhances considerably

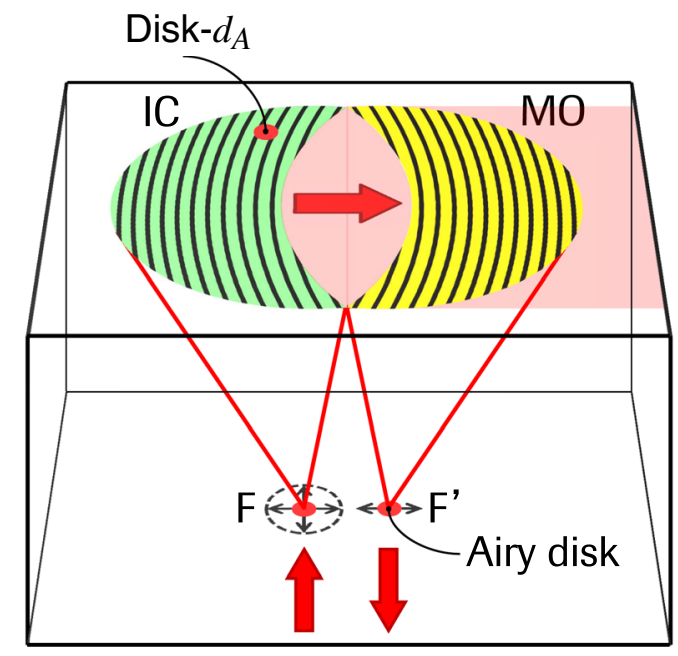

FIG. 3. Perspective view of the imaging system with optically conjugated foci $\mathrm{F}$ and $\mathrm{F}^{\prime}$. Both the area of the input coupler (IC) (green region) and the area of the mologram (MO) (yellow region) are restricted to lunes, i.e., a lunar crescent. 
the local light intensity that impinges on the mologram on the waveguide surface. The enhancement factor depends on the diameter $D$ of the IC, its coupling efficiency, and on the optical parameters of the waveguide [32]. We consider a waveguide of high refractive index (e.g., $\mathrm{Ta}_{2} \mathrm{O}_{5}$, $\left.n_{f}=2.117, \lambda=635 \mathrm{~nm}\right)$ with thickness $t_{f}=145 \mathrm{~nm}$ on a glass substrate with refractive index $n_{s}=1.521$ covered with an aqueous medium with refractive index $n_{0}=1.33$. The effective thickness of the waveguide for the TE mode $t_{\text {eff }}=t_{f}+\Delta z_{f, s}+\Delta z_{f, 0}$ is $329 \mathrm{~nm}$ with the penetration depth into the cover medium $\Delta z_{f, 0}=82 \mathrm{~nm}$. A conservative estimate for the coupling efficiency of the beam impinging on the IC is approximately $30 \%$; this value corresponds to a lune-shaped IC with diameter $D=$ $0.4 \mathrm{~mm}$ and a canonic surface-relief grating at the boundary between the waveguide and the substrate with a modulation depth of approximately $12 \mathrm{~nm}$ [32]. The stated parameters yield an enhancement factor of approximately 100 for the light intensity impinging on the mologram. Coupling of the incident beam to the waveguide gives rise to local dark-field illumination of the mologram on the surface.

The curved grating lines of the IC and the mologram with focal length $f$ are defined through the equation

$$
\pm\left(x_{j}-\xi\right)=\frac{\lambda N\left(j_{0}+j\right)-\sqrt{n_{s}^{2}\left(N^{2}-n_{s}^{2}\right)\left(y_{j}^{2}+f^{2}\right)+\left(n_{s} \lambda\right)^{2}\left(j_{0}+j\right)^{2}}}{N^{2}-n_{s}^{2}},
$$

where $j$ is the index of the line $\left(x_{j}, y_{j}\right)$. The \pm sign in Eq. (4) goes with the plus sign for the mologram and with the minus sign for the IC. The distance between the foci $\mathrm{F}$ and $\mathrm{F}^{\prime}$ is $2 \xi . N=1.814$ denotes the effective refractive index of the TE mode of the $\mathrm{Ta}_{2} \mathrm{O}_{5}$ waveguide. $j_{0}$ denotes the number of wavelengths in the substrate that add up to the focal length $f$; thus, $f=j_{0}\left(\lambda / n_{s}\right)$. The lune-shaped mologram with diameter $D=400 \mu \mathrm{m}$ and focal length $f=500 \mu \mathrm{m}$ comprises approximately 600 lines; see Fig. 3 . The distance between adjacent lines at their crossings of the symmetry axis of the grating structure varies continuously from approximately 338 to $267 \mathrm{~nm}$ for values of $j$ between 62 and 663. To avoid the resonant case (second-order Bragg reflection) in the propagation path of the waveguide mode, the grating is omitted in the $U$-shaped central area of the IC and the mologram, where the magnitude of the coupling angle would be close to zero [32].

In focal molography, "diffracted light" from coherently assembled biomolecules is brought to interference in a diffraction-limited focus. The mologram diffracts and focuses the phase contrast of the coherently assembled molecules into an Airy disk. The diameter $d_{A}$ of the Airy disk is given by Abbe's formula $d_{A}=(\lambda f) /\left(n_{s} D\right)=$ $\lambda /(2 \mathrm{~N}$.A. $)$, where N.A. $=\left(n_{s} D\right) /(2 f)$ is the numerical aperture of the mologram. For simplicity, the N.A. of the mologram is defined in analogy to the N.A. of a microscope objective; it does not take into account the lacking half in the lune-shaped mologram. The properties of the light in the focus of the mologram are used for distinct detection of refractive-index contrast in the proximity of the coherently assembled biomolecules from which information on the interaction of the immobilized biomolecules with molecules in a liquid or gaseous sample is obtained. The noncoherent surroundings of the coherently assembled biomolecules consisting of freely diffusing solvent and solute molecules (e.g., ions, detergents, plasma proteins, enzymes, cofactors, chaperons, ligand molecules) as well as changes in temperature do not contribute to the coherent signal in the focus of the mologram.

\section{Light-induced synthesis of the coherent pattern of molecules on a monolithic substrate}

We briefly describe here one possible route for creating the coherent pattern of receptor molecules on the sensor surface; see Fig. 4 and the Supplemental Material [33].

Nanopatterning, e.g., interference lithography [34], or high-resolution-imaging lithography, can be used to create a coherent pattern of receptor molecules with submicron resolution, which is a synthetic phase hologram that generates a diffraction-limited light wave out of a coherent pattern of molecules. State-of-the-art photolithography is suited for obtaining both submicron resolution and spatial coherence over the diameter $D$ of the mologram; both features are required for the site-directed immobilization of the receptor molecules in the mologram.

The synthesis of the molographic nanopattern is preferentially accomplished within a thin hydrogel layer. The hydrogel layer has three key properties. First, before the application of biolithography to the layer, the layer on the sensor surface must be monolithic and homogeneous, both in terms of its chemical and its optical properties. Second, the layer carries linker molecules with a terminal functional group for immobilization of the receptor. The terminal group on the linker is initially protected by a photolabile protecting group for light-induced immobilization of the receptor. Finally, the layer on the sensor surface has to be resistant to nonbiospecific protein adsorption [35-37].

The hydrogel layer on the sensor surface may be coupled to the surface of the $\mathrm{Ta}_{2} \mathrm{O}_{5}$ waveguide through an intermediate self-assembled monolayer (SAM) [37] on the tantalum-oxide surfaces [38]. The SAM on the $\mathrm{Ta}_{2} \mathrm{O}_{5}$ waveguide may consist of alkyl phosphate with a suitable functional group at the terminal position of the alkyl 
(a)

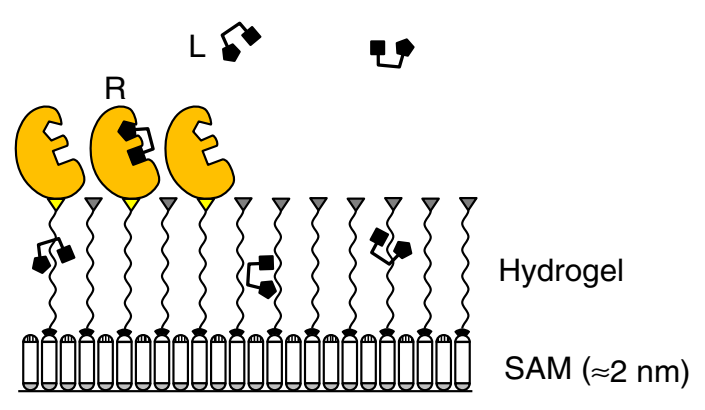

(b)

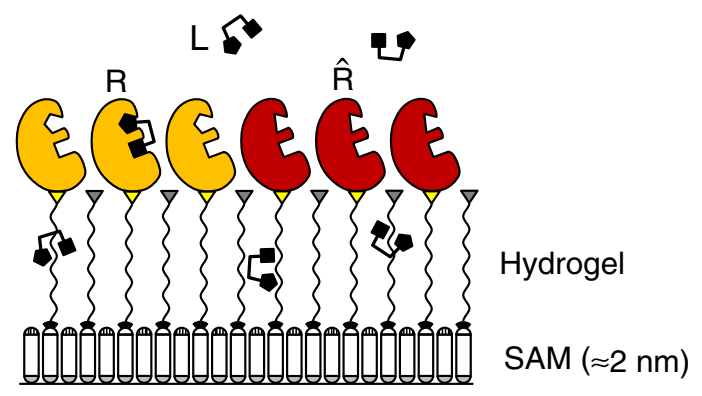

FIG. 4. Only one local period of the mologram consisting of one "ridge" and one "groove" is shown in Figs. 4(a) and 4(b). (a) Nonbackfilled mologram. (b) Backfilled mologram: The grooves between the receptor molecules $\mathrm{R}$ are filled with an unreceptive (e.g., point-mutated) mimetic receptor $\hat{\mathrm{R}}$ (red molecules). $\hat{R}$ does not recognize the ligand $L ; \hat{R}$ is unreceptive for $\mathrm{L}$. $\mathrm{L}$ does not bind to $\hat{\mathrm{R}}$. (a), (b) The ligand L (black molecules) is recognized by the receptor $\mathrm{R}$ (yellow molecules) and binds to the receptor R. Ligand molecules that bind nonbiospecifically to the hydrogel or to the SAM on the sensor surface are homogeneously distributed across the sensor surface; these molecules do not contribute to the coherent signal in the focus of the mologram.

phosphate for coupling of the hydrogel to the SAM [37,38]. The phosphate heads of the self-assembled alkyl phosphate provide the binding structure of the SAM layer to $\mathrm{Ta}_{2} \mathrm{O}_{5}$ [38].

The structure of one local period of the mologram on the sensor surface is not drawn to scale in Fig. 4: The hydrogel layer carrying the linkers to which the receptor molecules are immobilized may be much thicker than the SAM on the waveguide. The immobilized receptor molecules may be embedded within the hydrogel. The lines of higher refractive index in the mologram are denoted as "ridges," while the lines of lower refractive index between the ridges are denoted as "grooves." The immobilized receptor molecules are distributed in the ridges of the mologram. The grooves between the ridges might consist of hydrogel (nonbackfilled mologram) or of backfilled grooves (backfilled mologram). Ligand molecules and other biological constituents of the aqueous sample medium can freely diffuse through the hydrogel on the sensor surface $[16,17,36]$.

Sensitive photochemistry for the deprotection of functional groups on linker molecules by photolysis is readily available [39-43]. The linker in the hydrogel is connected at one end to the hydrogel, while the other terminal group on the linker is protected by a photolabile protecting group for light-induced immobilization of the receptor within the hydrogel. Straightforward strategies based on the use of a photocleavable protecting group offer great potential for the control of biological organization at micrometer and nanometer length scales [41]. Suitable linker chemistry and photochemistry with excellent resistance to nonbiospecific protein adsorption are readily available for the lithographic synthesis of molograms for diverse applications [41-43].

The key steps of a possible route for creating the coherent pattern of receptor molecules in a homogeneous hydrogel layer on the sensor surface are outlined and illustrated in the Supplemental Material [33].

In Fig. 4, the binding of a ligand to the receptor is shown in (a) for a nonbackfilled mologram and in (b) for a backfilled mologram. In a backfilled mologram, only ligand molecules that are bound to receptor molecules contribute to the light intensity in the focus of the mologram. Backfilling of the mologram leads to vanishing diffraction intensity in the focus of the ligand-free mologram. Ideal backfilling is achieved with a perfect mimetic of the receptor that can be obtained, for example, through a point mutation in the binding pocket of the investigated receptor protein.

We propose the term "activation lithography" for lightinduced synthesis of the mologram in a homogeneous layer on a monolithic substrate. The word "activation" indicates that the lithographic process is used for the activation of immobilization sites on the sensor surface. Activation lithography is used to direct the immobilization of biomolecules into a mologram that may be embedded in a hydrogel. Activation lithography does not affect the optical properties of the layered structure on the sensor surface.

When working with thoroughly optimized "proteinresistant" surfaces, the nonbiospecific adsorption of proteins from serum samples can be as low as $20-50 \mathrm{pg} / \mathrm{mm}^{2}$ $[24,35]$. In surface-capture assays, nonbiospecific protein adsorption to the surface of the sensor chip can easily reach $100 \mathrm{pg} / \mathrm{mm}^{2}$ or more [36]. Because molecules that nonbiospecifically adsorb to the layer on the sensor surface are homogeneously distributed across the layer, the nonbiospecific adsorption of proteins and other molecules on the sensor surface does not contribute to the light intensity in the diffraction focus of the mologram.

\section{Sensitivity of focal molography on a thin-film optical waveguide}

In the following, we derive analytical formulas that estimate the detection signal and the expected sensitivity of the focal molography scheme depicted in Fig. 3 in terms of known parameters. From the leakage of the TE mode induced by the refractive-index modulation in the mologram, we calculate the power $P_{\text {diff }}$ diffracted in the 
half-spaces above and below the surface, respectively. The leakage power tends to divide equally between the two halfspaces [32]. With the leakage parameter $A_{\text {molo }}$ for the TE mode for a canonic grating with alternating rectangular cells of lower (in the grooves) and higher (in the ridges) refractive index, we obtain the following for a small power leakage across the area of a nonbackfilled mologram [32]:

$$
\frac{P_{\text {diff }}}{P_{w g}}=A_{\text {molo }} \frac{\pi D}{4}=\frac{\left(n_{f}^{2}-N^{2}\right)\left(n_{m}{ }^{2}-n_{0}^{2}\right)^{2}}{N\left(n_{f}^{2}-n_{0}{ }^{2}\right)} \frac{\Lambda t_{m}{ }^{2}}{\lambda^{3} t_{\text {eff }}} \frac{\pi D}{2},
$$

where $t_{m}$ is the height of the rectangular cells in the canonic mologram. We assume $t_{m} \leq \lambda / 20$, so the parabolic region of $A_{\text {molo }}$ applies [32]. $P_{w g}$ is the power of the TE mode impinging on the mologram. In Eq. (5), the field distribution of the guided mode is taken as invariant with $y$ for $|y|<D / 2$ and 0 for $|y|>D / 2$. The higher refractive index $n_{m}$ in the ridges is given by $n_{m}=n_{0}+(d n / d c) c_{R}$, where $c_{R}$ denotes the concentration of the receptor in the ridges. $\Lambda=\lambda / n_{m}$ is the wavelength in the ridges [32]. For low $c_{R}$, we have $\Lambda \approx \lambda / n_{0}$. Relation (5) can be expressed in the well-known differential sensitivity $\partial N / \partial n_{0}$ of the refractometric thin-film waveguide biosensor [44]

$$
\begin{gathered}
\frac{P_{\text {diff }}}{P_{w g}}=\frac{\partial N}{\partial n_{0}} \frac{\left(n_{m}^{2}-n_{0}^{2}\right)^{2}}{n_{0}} \frac{\Lambda t_{m}^{2}}{\lambda^{3} \Delta z_{f, 0}} \frac{\pi D}{2} \\
\approx \frac{\partial N}{\partial n_{0}} \frac{8 \pi n_{0} \Lambda D}{\lambda^{3} \Delta z_{f, 0}}\left(\frac{d n}{d c}\right)^{2} \Gamma_{\Delta}^{2}, \\
\text { with } \quad \frac{\partial N}{\partial n_{0}}=\frac{n_{0}}{N} \frac{n_{f}^{2}-N^{2}}{n_{f}^{2}-n_{0}^{2}} \frac{\Delta z_{f, 0}}{t_{\text {eff }}} .
\end{gathered}
$$

The diffracted power $P_{\text {diff }}$ goes with the square of the surface mass-density modulation $\Gamma_{\Delta}=\left(t_{m} c_{R}\right) / 2$ in the mologram. The refractive-index modulation $\left(n_{m}^{2}-n_{0}^{2}\right)$ in Eq. (5) arises from regular density fluctuations of the molecules in the mologram and is given by the formula

$$
\left(n_{m}^{2}-n_{0}^{2}\right)=\left(2 n_{0}+\frac{d n}{d c} c_{R}\right) \frac{d n}{d c} c_{R} \approx 2 n_{0} \frac{d n}{d c} c_{R} .
$$

The approximations in Eqs. (6) and (8) are valid for small $c_{R}$. For the stated waveguide parameters, $\partial N / \partial n_{0}$ equals 0.080 for the TE mode. We compare the power density of diffracted light on the Airy disk $P_{\text {diff }}$ (Airy) to the power density irradiated on the input coupler. We relate $P_{\text {diff }}$ (Airy) $=P_{\text {diff }}$ from (6) to the power $P_{m}\left(\right.$ Disk- $\left.d_{A}\right)$ incident on a disk with diameter $d_{A}$ in close proximity to the plane of the mologram. The "Disk- $d_{A}$ " is located above or below the input coupler outside the intense field in the waveguide (see Fig. 3)

$$
\frac{P_{\text {diff }}(\text { Airy })}{P_{m}\left(\text { Disk- } d_{A}\right)} \approx \eta \frac{\partial N}{\partial n_{0}} \frac{\left.8 \pi n_{0} \Lambda D^{3} \text { (N.A. }\right)^{2}}{\lambda^{5} \Delta z_{f, 0}}\left(\frac{d n}{d c}\right)^{2} \Gamma_{\Delta}^{2} \text {. }
$$

The expression for $P_{\text {diff }}$ (Airy) from Eq. (6) that was inserted on the right side of Eq. (9) was divided by 4 to account for the lacking half in the lune-shaped mologram. For $n_{0}=1.33, n_{s}=1.521, \lambda=635 \mathrm{~nm}, \Lambda \approx \lambda / n_{0}=477 \mathrm{~mm}$, $D=0.4 \mathrm{~mm}, f=0.5 \mathrm{~mm}$ [N.A. $=\left(n_{s} D\right) /(2 f)=0.61$ ], $\Delta z_{f, 0}=82 \mathrm{~nm}, d n / d c=0.182 \mathrm{ml} / \mathrm{g}$, coupling efficiency of input coupler $\eta=0.3$, and $\partial N / \partial n_{0}=0.080$, we obtain from Eq. (9) for $P_{\text {diff }}$ (Airy) $/ P_{m}\left(\right.$ Disk- $\left.d_{A}\right)$ the values of approximately $2.3 \times 10^{-3}$ for 1-pg protein and approximately 2300 for 1 -ng protein in the mologram, respectively. The diffracted power $P_{\text {diff }}$ (Airy) irradiated by the coherently assembled molecules in the mologram is concentrated in a focal spot $\mathrm{F}^{\prime}$ with diameter $d_{A}=(\lambda f) /\left(n_{s} D\right) \approx 0.52 \mu \mathrm{m}$. The ratio $P_{\text {diff }}($ Airy $) / P_{m}\left(\right.$ Disk- $\left.d_{A}\right)$ in Eq. (9) reaches the value 1 for approximately 21-pg protein in the mologram with $D=0.4 \mathrm{~mm}$. This very small amount of coherently assembled biological matter irradiates power density on the Airy disk in the focus of the mologram that equals the power density of the beam impinging on the input coupler; see Fig. 3. For comparison: One closely packed monolayer of water molecules on the surface area of the mologram with $D=0.4 \mathrm{~mm}$ contains approximately 39-pg water [8].

The calculated values $P_{\text {diff }}$ (Airy) $/ P_{m}\left(\right.$ Disk- $\left.d_{A}\right)$ obtained from Eq. (9) for the mologram on the waveguide are about 40 times larger than the values $P_{\text {scat }}($ Airy $) / P_{L}\left(\right.$ Disk- $\left.d_{A}\right)$ obtained from Eq. (3) for the coherent scattering system on the ellipsoid of revolution in a homogeneous medium $n_{0}$ that contains the same amount of biological matter as the mologram on the waveguide; see Figs. 1 and 3. The results obtained from Eqs. (3) and (9) are consistent with the intensity enhancement of the light impinging on the mologram on the thin-film optical waveguide that is caused by resonant coupling of the incident beam to the waveguide. The consistency of the two results is given within the expected uncertainties stemming from the approximations used in the two calculations on the different but physically related optical configurations, i.e., the power scattered by the coherent scattering system in Sec. II A and the power diffracted by the mologram on the waveguide in Sec. II D.

The light propagating in the waveguide is subjected to diffraction by the coherently assembled molecules on the waveguide and to scattering by small deviations from the perfect straightness of the waveguide walls [45]. The $\mathrm{Ta}_{2} \mathrm{O}_{5}$ waveguide is assumed to be prepared by ion beam sputtering. It has a propagation loss of approximately $0.8 \mathrm{~dB} / \mathrm{cm}$ for the TE mode when covered with an aqueous medium. We assume that the propagation loss in the waveguide is entirely due to scattering of the propagating mode and that the scattered light is evenly distributed over the solid angle $4 \pi[45]$.

We restrict the detection of the irradiance incident on the Airy disk to the aperture of the mologram. The ratio 
between the diffracted power $P_{\text {diff }}$ (Airy) and the scattered power $P_{\text {scat }}($ Airy) incident on the Airy disk is given by

$$
\begin{aligned}
\frac{P_{\text {diff }}(\text { Airy })}{P_{\text {scat }}(\text { Airy })} & =\frac{A_{\text {molo }}}{A_{\text {scat }}} \frac{2 n_{s}{ }^{2} D^{2}}{\lambda^{2}} \\
& =\frac{\partial N}{\partial n_{0}} \frac{64 n_{0} n_{s}{ }^{2} \Lambda D^{2}}{\lambda^{5} \Delta z_{f, 0} A_{\text {scat }}}\left(\frac{d n}{d c}\right)^{2} \Gamma_{\Delta}{ }^{2} .
\end{aligned}
$$

For $n_{0}=1.33, n_{s}=1.521, \lambda=635 \mathrm{~nm}, \Lambda \approx \lambda / n_{0}=$ $477 \mathrm{~mm}, D=0.4 \mathrm{~mm}, \Delta z_{f, 0}=82 \mathrm{~nm}$, leakage parameter $A_{\text {scat }}=10^{-2} \mathrm{~mm}^{-1}(0.8-\mathrm{dB} / \mathrm{cm}$ propagation loss $), d n / d c=$ $0.182 \mathrm{ml} / \mathrm{g}$, and $\partial N / \partial n_{0}=0.080$, we obtain for $P_{\text {diff }}$ (Airy) $/ P_{\text {scat }}$ (Airy) the values 30 for 1 -pg protein and $3 \times 10^{7}$ for 1 -ng protein in the thin mologram, respectively. The ratio $P_{\text {diff }}$ (Airy) $/ P_{\text {scat }}$ (Airy) in Eq. (10) reaches the value 1 for approximately 180 -fg protein in the mologram with $D=0.4 \mathrm{~mm}$. This minuscule amount of coherently assembled biological matter irradiates power density on the Airy disk in the focus of the mologram that equals the power density of the noncoherent irradiance of scattered light impinging on the focal plane of the mologram.

The smallest possible refractive-index contrast of the ligand-free mologram can be attained by backfilling of the grooves between the ridges with an unreceptive (e.g., pointmutated) mimetic receptor $\hat{R}$. The binding of ligand molecules to the receptor $\mathrm{R}$ in the ridges of the canonic mologram changes the surface mass-density modulation $\Gamma_{\Delta}$ in Eqs. (9) and (10), according to

$$
\begin{aligned}
\Gamma_{\Delta}^{2}= & {\left[\Gamma_{\Delta+}\left(c_{R}\right)-\Gamma_{\Delta-}\left(c_{\hat{R}}\right)+\Gamma_{\Delta+}\left(c_{L}\right)\right]^{2} } \\
= & {\left[\Gamma_{\Delta+}\left(c_{R}\right)-\Gamma_{\Delta-}\left(c_{\hat{R}}\right)\right]^{2}+2\left[\Gamma_{\Delta+}\left(c_{R}\right)\right.} \\
& \left.-\Gamma_{\Delta-}\left(c_{\hat{R}}\right)\right] \Gamma_{\Delta+}\left(c_{L}\right)+\left[\Gamma_{\Delta+}\left(c_{L}\right)\right]^{2} \approx\left[\Gamma_{\Delta+}\left(c_{L}\right)\right]^{2} .
\end{aligned}
$$

$\Gamma_{\Delta+}$ and $\Gamma_{\Delta-}$ denote the contribution of the ridges and the contribution of the grooves to the surface mass-density modulation in the mologram, respectively. $c_{\hat{R}}$ denotes the concentration of the inactive (e.g., point-mutated) receptor $\hat{\mathrm{R}}$ in the grooves. $c_{L}$ denotes the concentration of the ligand $\mathrm{L}$ in the ridges. For a nonbackfilled mologram, $c_{\hat{R}}=\Gamma_{\Delta-}=0$. The approximation in Eq. (11) applies for perfect backfilling. Formula (11) applies to partial and full receptor occupancy.

The noncoherent irradiance generated by scattering of the propagating mode at small deviations of the waveguide from perfect straightness is evenly distributed in the focal plane of the mologram [45]. The Airy disk is embedded in this background. The measured noncoherent irradiance in the focal plane outside the Airy disk can be subtracted from the measured power density in the Airy disk. The resulting value can be used for the accurate quantification of a molecular recognition and binding interaction of the coherently assembled receptor in the mologram with a freely diffusing ligand.
The calculation of the result (10) assumes that the radiation loss caused by the broad power spectrum of the small irregularities of the waveguide is evenly distributed over the solid angle $4 \pi$. Actually, the Fourier components responsible for forward and backward scattering contribute more to the total radiation loss of the propagating mode than Fourier components causing scattering in other directions [45]. The preferential loss behavior of forward and backward scattering is not taken into account in Eq. (10). Thus, the result in Eq. (10) underestimates the amount of coherent irradiance in the diffraction focus of the mologram in relation to the scattered irradiance in the focal plane of the mologram. The ratio of the power density $P_{\text {diff }}$ (Airy) of the diffracted light in the focus of the mologram divided by the power density $P_{\text {scat }}$ (Airy) resulting from scattering of the guided mode is somewhat higher than the value given in Eq. (10).

The mologram is sensed by the evanescent field of the guided mode extending from the waveguiding film into the aqueous cover medium. The results (6), (9), and (10) are valid for thin molograms $\left(t_{m} \leq \lambda / 20 \approx 32 \mathrm{~nm}\right.$, i.e., in the parabolic region of $A_{\text {molo }}$ vs $t_{m}$ ) and also for very thin molograms $\left(t_{m}<1 \AA\right)$, where $t_{m}$ resembles the averaged thickness of a submonolayer. The distribution of the ligand molecules perpendicular to the surface only slightly changes $P_{\text {diff }}$ as long as the mologram is thin $\left(t_{m} \leq \lambda / 20\right)$. For a given waveguide, the sensitivity of focal molography in terms of the lowest detectable surface mass-density modulation $\Gamma_{\Delta}$ decreases for thicker molograms. For molograms with $t_{m}>\Delta z_{f, 0}$, the contribution from molecules situated beyond the penetration depth $\Delta z_{f, 0}$ of the evanescent field on the leakage parameter $A_{\text {molo }}$ decreases rapidly.

Expressions analogous to Eqs. (5), (6), (7), (9), and (10) can be derived for the fundamental TM mode of the considered waveguide and for the higher modes of thicker waveguides $[32,44]$. The influence of the waveguide parameters on the sensitivities of focal molography for the TE and TM modes of the waveguide can be derived from the analysis of the differential sensitivities $\partial N / \partial n_{0}$ of the refractometric thin-film waveguide biosensor given in Ref. [44].

For relatively thick molograms having a thickness in the range of the penetration depth of the evanescent field, the sensitivity of focal molography may be enhanced by using a reverse-symmetry thin-film waveguide with a relatively low refractive index [46].

\section{E. Readout of the mologram through a bifocal laser-scanning microscope}

Readout of the mologram is achieved by a confocal laserscanning microscope with some add-on components in its beam path for the directional separation of incoupling and outcoupling. The add-ons make the confocal bifocal: Two beam stops (BSs), complementary to each other, restrict 
half of the cross sections of the incident and the detection beam; see Fig. 5.

The incident laser beam is coupled to the waveguide via the objective and the focal IC, located alongside each mologram. A scan of $\mathrm{F}$ in the $x$ direction adjusts the incident beam to the highest input coupling; see Fig. 3. The light decoupled by the mologram travels back via the scanner-i.e., the beam motion is neutralized again-and then passes through the beam splitter and is focused onto the optical plane of the pinhole (PH). The PH is adjusted in the $x$ direction to maximize the light intensity on the detector. The detector behind the pinhole measures the light intensity in the focus of the mologram. The focal plane of the objective is adjusted to the plane parallel to the waveguide that contains the two foci $\mathrm{F}$ and $\mathrm{F}^{\prime}$. Scanning of the two foci $\mathrm{F}$ and $\mathrm{F}^{\prime}$ in three dimensions is required to measure the light intensity in the focus of the mologram at its maximum in terms of both input and output coupling. Two dimensions of the scan come from the fast scanner in the microscope and the third from the slide of the pinhole. The motion of the $\mathrm{PH}$ to a position away from $\mathrm{F}^{\prime}$ (or scanning of $\mathrm{F}$ in the $y$ direction) can be used to measure the intensity of the noncoherent background and to deduct it from the intensity in $\mathrm{F}^{\prime}$.

Present-day confocal laser-scanning microscopes achieve multisite high-speed imaging through high-speed scanning with acousto-optic deflectors [47], which can generate fast discontinuous scanning patterns [47]. The diffractive nature of the acousto-optic deflection complicates the descanning of the nonmonochromatic light emitted from the fluorescent samples investigated by normal confocal laser-scanning microscopes. In the conceived bifocal laser-scanning setup, however, descanning is not a problem because the readout of the mologram on the

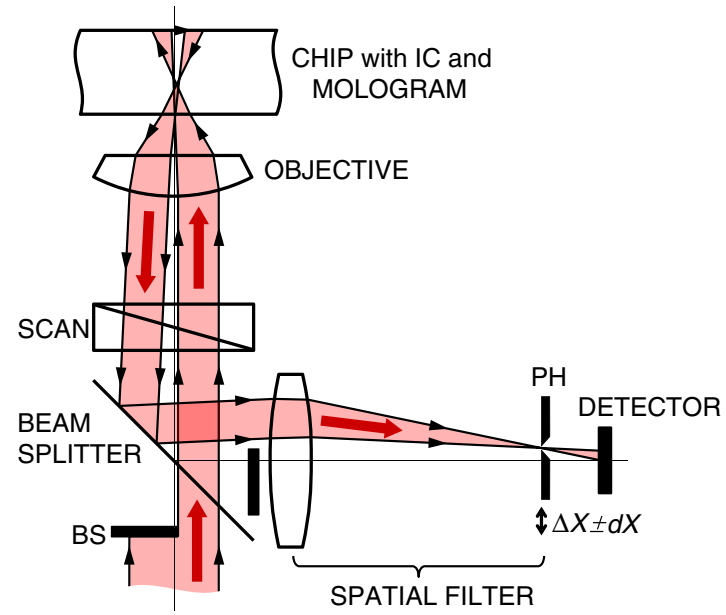

FIG. 5. Readout of the mologram through a bifocal laserscanning microscope: The PH in front of the detector is adjusted to the optically conjugated position to $\mathrm{F}^{\prime}$; adjustment of the $\mathrm{PH}$ to the optically conjugated position $\mathrm{F}^{\prime}$ makes the modified confocal laser-scanning microscope "bifocal." sensor chip is monochromatic. Thus, the descanning of the outcoupled beam from the mologram in the acousto-optic deflector is not hindered by the diffractive nature of the acousto-optic deflection.

Acousto-optic deflection permits fast scanning and descanning of the monochromatic laser beams coupled into and out of the mologram with no macroscopically moving parts. The combined object scanning and beam scanning under the bifocal laser-scanning microscope is expected to be well suited for the readout of high-density mologram arrays on chips with the typical size of a microscope slide.

\section{CONCLUSIONS AND FUTURE DIRECTIONS}

In summary, we have a promising new analytical method-focal molography - that is expected to enable gentle, sensitive, and robust label-free probing of biomolecular interactions in real time. Biomolecules may be immobilized in a distinct submicron pattern within a thin hydrogel layer on the surface of a chip. Molecules that interact with the biomolecules in the thin layer get ordered. The induced order is comprehensively detected through diffracted light; direct light is shielded off. Hence, molecular interactions can be made visible by the measurement of changes of the phase contrast in the nanoenvironment of the immobilized biomolecules. In contrast to the currently used refractometric optical biosensors (SPR and optical waveguide sensors), focal molography is not hampered by the noncoherent surroundings of the coherently assembled molecules consisting of freely diffusing solvent and solute molecules.

Focal molography may evolve into an enabling method for the discovery of yet unknown biomolecular interactions and for the identification of molecules that interfere with protein-protein interactions. The biospecificity of the method can be enhanced considerably through the correlated detection of multiple recognitions of the targeted molecule (the analyte) by different interaction partners immobilized in multiplexed or superimposed molograms on the same surface.

The immobilization of small- and medium-size molecules that bind or may bind to proteins (known drugs, bioactive small molecules, synthetic peptide structures, and bioactive natural products) in an array of molograms to a very thin layer of affinity resin on a chip represents a promising potential application of focal molography for target identification by affinity capture through chemical proteomics in the pharmaceutical sciences $[48,49]$. In this particular application of focal molography, the smallmolecule ligand is immobilized to the thin layer on the chip and the affinity of a much larger receptor protein to the small ligand is investigated through molographic readout. The problem is that many high-abundance proteins bind nonbiospecifically to the bare resin [48]. At this time, it is impossible to measure accurately the binding affinity of a 
small molecule to its target protein without knowing the identity of the target protein [48]. This deficiency holds especially true for low-abundance target proteins [48]. Because focal molography is insensitive to the nonbiospecific binding of high-abundance proteins in the investigated cell extract to the bare resin, the new method may enable "bottom-up pull-down assays with in situ readout of the binding kinetics" of proteins that are captured by small- and medium-size immobilized binders in arrays of molograms.

Biomedical diagnostics based on molecular recognition involves the detection of low concentrations of known analytes with relatively high molecular weight (e.g., a protein, a peptide, or an oligonucleotide). Such analytesthey are also termed large-molecule diagnostic biomarkerscan be simultaneously recognized and bound by two complementary binding partners that interact with the analyte via complementary binding sites on the analyte. The second binder complements the first and carries a label. The immobilized binder captures the targeted analyte. The complementary binder binds via a complementary binding site to the analyte and forms a sandwich complex on the sensor surface. The sandwich complex consists of the immobilized binder, the analyte, and the complementary binder carrying the label [50]. The presence of the label on the sensor surface indicates the formation of a sandwich complex on the surface that, in turn, is an indication of the presence of the analyte in the investigated sample [50]. For refractometric optical biosensors, the labeling of the complementary binder to the targeted analyte is preferentially achieved with a high-refractive-index label such as a gold nanoparticle [24].

The focal molography method described in this paper can - in principle - also be applied to sandwich assays with a refractive-index label that enhances the refractive-index change associated with the formation of the sandwich complex on the sensor surface. Because focal molography detects only the coherently assembled nanoparticles on the surface of the chip-i.e., those particles that became ordered through the formation of the sandwich complex consisting of the immobilized binder, the analyte, and the complementary binder carrying the gold nanoparticle-a nanoparticle focal molography assay can be "separation free," i.e., not requiring a washing step for separation of the bound and the free complementary binders in the boundary layer on the sensor surface before detection of the sandwich complex.

Methods for identifying and exploiting biomolecular interactions rely on two experimental steps: identification of intermolecular interaction partners by a variety of methods and high-resolution structure analysis of the complex formed by the bound partner molecules. The second step is mainly achieved by the x-ray crystallography of highly ordered crystals prepared by crystallogenesis of the binding partners; see, e.g., Refs. [51,52]. Even though the crystalline state of biomolecules is hardly ever encountered in nature, it has proven to be pivotal for our understanding of the interplay between the structure and the function of biomolecules [51,52]. The investigation presented in this paper was inspired by a scientific question: Besides densely packed protein crystals, is there a more "open" but still regular (i.e., coherent) man-made assembly of molecules that could facilitate the elucidation of biomolecular interactions? An "open coherent assembly" in this context means that the assembly has space between the molecules that can be accessed by molecules in a free solution. One main point to be considered is the absence of steric hindrance within the mologram. The mologram is an open and coherent assembly of immobilized molecules; it avoids steric hindrance that occurs from overcrowding of the molecules in the coherent assembly.

Focal molography, precisely because it utilizes the power of coherent and diffractive methodology, is highly sensitive and robust. Changes of the bulk refractive index in the thin layer on the sensor surface are noncoherent and do not contribute to the measured focal signal. Focal molography provides intrinsic discrimination of uncontrolled background signals that are due to nonbiospecific interactions in the boundary layer containing the coherently assembled biomolecules.

The realization of focal molography and experimental results will be presented in subsequent publications.

\section{ACKNOWLEDGMENTS}

The author acknowledges with gratitude Janos Vörös, Laboratory of Biosensors and Bioelectronics, ETH-Zurich, for invigorating discussions on biosensors and critical comments on the manuscript. The author would like to thank Klaus-Peter Stengele, Roche Diagnostics, for fruitful discussions on linker chemistry and photolabile building blocks for the photolithographic formation of high-quality biochips. The author thanks David W. Banner for help with manuscript preparation.

[1] F. Zernike, How I Discovered Phase Contrast, Science 121, 345 (1955).

[2] K. Michel, Das Phasenkontrastverfahren und seine Eignung für zytologische Untersuchungen, Naturwissenschaften 37, 52 (1950).

[3] P. Marquet, B. Rappaz, P. J. Magistretti, E. Cuche, Y. Emery, T. Colomb, and C. Depeursinge, Digital Holographic Microscopy: A Noninvasive Contrast Imaging Technique Allowing Quantitative Visualization of Living Cells with Subwavelength Axial Accuracy, Opt. Lett. 30, 468 (2005).

[4] P. Jourdain, D. Boss, B. Rappaz, C. Moratal, M.-C. Hernandez, C. Depeursinge, P. Julius Magistretti, and P. Marquet, Simultaneous Optical Recording in Multiple Cells by Digital Holographic Microscopy of Chloride Current Associated to Activation of the Ligand-Gated Chloride Channel GABA(A) Receptor, PLoS One 7, e51041 (2012). 
[5] R. Karlsson, A. Michaelsson, and L. Mattsson, Kinetic Analysis of Monoclonal Antibody-Antigen Interactions with a New Biosensor Based Analytical System, J. Immunol. Methods 145, 229 (1991).

[6] M. Malmqvist, Biospecific Interaction Analysis Using Biosensor Technology, Nature (London) 361, 186 (1993).

[7] M. Nirschl, F. Reuter, and J. Vörös, Review of Transducer Principles for Label-Free Biomolecular Interaction Analysis, Biosensors 1, 70 (2011).

[8] R. Hoyer, C. Mangold, C. Fattinger, M. Heming, B. Danielzik, J. Otto, and M. Lohmeyer, Highly Sensitive Interfacial Mass Detection Using Ultracompact Waveguiding Films, Appl. Phys. Lett. 64, 2791 (1994); Erratum: "Highly Sensitive Interfacial Mass Detection Using Ultracompact Waveguiding Films,” 65, 377 (1994).

[9] W. Lukosz, Principles and Sensitivities of Integrated Optical and Surface Plasmon Sensors for Direct Affinity Sensing and Immunosensing, Biosensors Bioelectronics 6 , 215 (1991).

[10] M. A. Cooper, Optical Biosensors in Drug Discovery, Nat. Rev. Drug Discovery 1, 515 (2002).

[11] R. Karlsson and R. Stahlberg, Surface Plasmon Resonance Detection and Multisport Sensing for Direct Monitoring of Interactions Involving Low-Molecular-Weight Analytes and for Determination of Low Affinities, Anal. Biochem. 228, 274 (1995).

[12] H. Nordin, M. Jungnelius, R. Karlsson, and O. P. Karlsson, Kinetic Studies of Small Molecule Interactions with Protein Kinases Using Biosensor Technology, Anal. Biochem. 340, 359 (2005).

[13] M. J. Cannon et al., Comparative Analyses of a Small Molecule/Enzyme Interaction by Multiple Users of Biacore Technology, Anal. Biochem. 330, 98 (2004).

[14] M. A. Cooper, Label-Free Biosensors: Techniques and Applications (Cambridge University Press, Cambridge, England, 2009).

[15] J. A. Defeijter, J. Benjamins, and F. A. Veer, Ellipsometry as a Tool to Study the Adsorption Behavior of Synthetic and Biopolymers at Air-Water Interface, Biopolymers 17, 1759 (1978).

[16] S. Lofas and B. Johnsson, A Novel Hydrogel Matrix on Gold Surfaces in Surface Plasmon Resonance Sensors for Fast and Efficient Covalent Immobilization of Ligands, J. Chem. Soc. Chem. Commun., 1526 (1990).

[17] B. Johnsson, S. Löfas, and G. Lindquist, Immobilization of Proteins to a Carboxymethyldextran-Modified Gold Surface for Biospecific Interaction Analysis in Surface Plasmon Resonance Sensors, Anal. Biochem. 198, 268 (1991).

[18] E. Stenberg, B. Persson, H. Roos, and C. Urbaniczky, Quantitative Determination of Surface Concentration of Protein with Surface Plasmon Resonance Using Radiolabeled Proteins, J. Colloid Interface Sci. 143, 513 (1991).

[19] N. Kovacs, D. Patko, N. Orgovan, S. Kurunczi, J. J. Ramsden, F. Vonderviszt, and R. Horvath, Optical Anisotropy of Flagellin Layers: In Situ and Label-Free Measurement of Adsorbed Protein Orientation Using OWLS, Anal. Chem. 85, 5382 (2013).

[20] Y. G. Tsay, C. I. Lin, J. Lee, E. K. Gustafson, R. Appelqvist, P. Magginetti, R. Norton, N. Teng, and D. Charlton, Optical
Biosensor Assay (OBA), Clinical Chemistry 37, 1502 (1991).

[21] X. Wang, M. Zhao, and D. D. Nolte, Molecular Layer Detection on a Diffractive Optical Balance, Opt. Lett. 37, 4098 (2012).

[22] J. Spinke, N. Oranth, C. Fattinger, H. Koller, C. Mangold, and D. Voegelin, The Bidiffractive Grating Coupler: Application to Immunosensing, Sensors Actuators B 39, 256 (1997).

[23] S. Kubitschko, J. Spinke, T. Bruckner, S. Pohl, and N. Oranth, Sensitivity Enhancement of Optical Immunosensors with Nanoparticles, Anal. Biochem. 253, 112 (1997).

[24] J. Martinez-Perdiguero, A. Retolaza, L. Bujanda, and S. Merino, Surface Plasmon Resonance Immunoassay for the Detection of the TNF $\alpha$ Biomarker in Human Serum, Talanta 119, 492 (2014).

[25] C.-L. Chang, G. Acharya, and C. A. Savran, In Situ Assembled Diffraction Grating for Biomolecular Detection, Appl. Phys. Lett. 90, 233901 (2007).

[26] R. P. Ekins, Ligand Assays: From Electrophoresis to Miniaturized Microarrays, Clinical Chemistry 44, 2015 (1998).

[27] T. M. Squires, R. J. Messinger, and S. R. Manalis, Making It Stick: Convection, Reaction and Diffusion in Surface-Based Biosensors, Nat. Biotechnol. 26, 417 (2008).

[28] D. Sinclair, Light Scattering by Spherical Particles, J. Opt. Soc. Am. 37, 475 (1947).

[29] R. Barer and S. Tkaczyk, Refractive Index of Concentrated Protein Solutions, Nature (London) 173, 821 (1954).

[30] H. Fischer, I. Polikarpov, and A. F. Craievich, Average Protein Density Is a Molecular-Weight-Dependent Function, Protein Sci. 13, 2825 (2004).

[31] C. Fattinger, The Bidiffractive Grating Coupler, Appl. Phys. Lett. 62, 1460 (1993).

[32] T. Tamir and S. T. Peng, Analysis and Design of Grating Couplers, Appl. Phys. 14, 235 (1977); Erratum: "Analysis and Design of Grating Couplers,” 21, 410(E) (1980).

[33] See Supplemental Material at http://link.aps.org/ supplemental/10.1103/PhysRevX.4.031024 for synthesis of a backfilled mologram by activation lithography on a monolithic substrate.

[34] T. J. Suleski, B. Baggett, W. F. Delaney, C. Koehler, and E. G. Johnson, Fabrication of High-Spatial-Frequency Gratings through Computer-Generated Near-Field Holography, Opt. Lett. 24, 602 (1999).

[35] S. Pasche, S. M. De Paul, J. Voros, N. D. Spencer, and M. Textor, Poly(L-lysine)-graft-poly(ethylene glycol) Assembled Monolayers on Niobium Oxide Surfaces: A Quantitative Study of the Influence of Polymer Interfacial Architecture on Resistance to Protein Adsorption by ToF-SIMS and In Situ OWLS, Langmuir 19, 9216 (2003).

[36] J.-F. Masson, T. M. Battaglia, J. Cramer, S. Beaudoin, M. Sierks, and K. S. Booksh, Reduction of Nonspecific Protein Binding on Surface Plasmon Resonance Biosensors, Anal. Bioanal. Chem. 386, 1951 (2006).

[37] E. Ostuni, L. Yan, and G. M. Whitesides, The Interaction of Proteins and Cells with Self-Assembled Monolayers of Alkanethiolates on Gold and Silver, Colloids Surf. B 15, 3 (1999). 
[38] D. M. Spori, N. V. Venkataraman, S. G. P. Tosatti, F. Durmaz, N. D. Spencer, and S. Zuercher, Influence of Alkyl Chain Length on Phosphate Self-Assembled Monolayers, Langmuir 23, 8053 (2007).

[39] S. Buhler, I. Lagoja, H. Giegrich, K. P. Stengele, and W. Pfleiderer, New Types of Very Efficient Photolabile Protecting Groups Based upon the [2-(2-nitrophenyl)propoxy] carbonyl (NPPOC) Moiety, Helv. Chim. Acta 87, 620 (2004).

[40] S. A. A. Ahmad, L. S. Wong, E. ul-Haq, J. K. Hobbs, G. J. Leggett, and J. Micklefield, Micrometer- and NanometerScale Photopatterning Using 2-Nitrophenylpropyloxycarbonyl-Protected Aminosiloxane Monolayers, J. Am. Chem. Soc. 131, 1513 (2009).

[41] S. A. A. Ahmad, L. S. Wong, E. ul-Haq, J. K. Hobbs, G. J. Leggett, and J. Micklefield, Protein Micro- and Nanopatterning Using Aminosilanes with Protein-Resistant Photolabile Protecting Groups, J. Am. Chem. Soc. 133, 2749 (2011).

[42] N. Laboria, R. Wieneke, and R. Tampe, Control of Nanomolar Interaction and In Situ Assembly of Proteins in Four Dimensions by Light, Angew. Chem., Int. Ed. Engl. 52, 848 (2013).

[43] K. A. Mosiewicz, L. Kolb, A. J. van der Vlies, M. M. Martino, P. S. Lienemann, J. A. Hubbell, M. Ehrbar, and M. P. Lutolf, In Situ Cell Manipulation through Enzymatic Hydrogel Photopatterning, Nat. Mater. 12, 1072 (2013).
[44] K. Tiefenthaler and W. Lukosz, Sensitivity of Grating Couplers as Integrated-Optical Chemical Sensors, J. Opt. Soc. Am. B 6, 209 (1989).

[45] D. Marcuse, Radiation Losses of Dielectric Waveguides in Terms of the Power Spectrum of the Wall Distortion Function, Bell Syst. Tech. J. 48, 3233 (1969).

[46] R. Horvath, H. C. Pedersen, and N. B. Larsen, Demonstration of Reverse Symmetry Waveguide Sensing in Aqueous Solutions, Appl. Phys. Lett. 81, 2166 (2002).

[47] P. Saggau, New Methods and Uses for Fast Optical Scanning, Curr. Opin. Neurobiol. 16, 543 (2006).

[48] S.-i. Sato, A. Murata, T. Shirakawa, and M. Uesugi, Biochemical Target Isolation for Novices: Affinity-Based Strategies, Chem. Biol. 17, 616 (2010).

[49] U. Rix and G. Superti-Furga, Target Profiling of Small Molecules by Chemical Proteomics, Nat. Chem. Biol. 5, 616 (2009).

[50] R. Maiolini and R. Masseyeff, A Sandwich Method of Enzymoimmunoassay. I. Application to Rat and Human Alpha-Fetoprotein, J. Immunol. Methods 8, 223 (1975).

[51] D. Banner and P. Hadvary, Crystallographic Analysis at $0-\AA$ Resolution of the Binding to Human Thrombin of 4 Active Site-Directed Inhibitors, J. Biol. Chem. 266, 20085 (1991).

[52] S. G. F. Rasmussen et al., Crystal Structure of the $\beta_{2}$ Adrenergic Receptor-Gs Protein Complex, Nature (London) 477, 549 (2011). 